\title{
Correlation of acidic mammalian chitinase expression with disease severity in patients with moderate/severe persistent allergic rhinitis
}

\author{
HAMID REZA SADEGHI ${ }^{1}$, ASHKAN PIRAYESH ${ }^{1,2}$, SHAGHAYEGH SHAHSAVAN ${ }^{1,2}$, \\ SOROUSH AMANI ${ }^{3}$ SAYED ASADOLLAH AMINI ${ }^{4}$ KEYHAN GHATREH SAMANI ${ }^{4}$, \\ FATEMEH DERIS
}

${ }^{1}$ Cellular and Molecular Research Center, Shahrekord University of Medical Sciences, Shahrekord, Iran

${ }^{2}$ Hematopoietic Stem Cell Transplantation and Cell Therapy Research Center, Taleghani Hospital, Shahid Beheshti University of Medical Science, Teheran, Iran

${ }^{3}$ Department of Internal Medicine, Shahrekord University of Medical Sciences, Shahrekord, Iran

${ }^{4}$ Clinical Biochemistry Research Center, Shahrekord University of Medical Sciences, Shahrekord, Iran

${ }^{5}$ Department of Biostatistics, Shahrekord University of Medical Sciences, Shahrekord, Iran

\begin{abstract}
Aim of the study: To assess the level of acidic mammalian chitinase (AMCase) expression and IL-8 in nasal inferior turbinate mucosa in patients with mild and moderate to severe allergic rhinitis (AR).

Material and methods: Participants in this case-control study were divided into three groups, including patients with moderate and severe persistent allergic rhinitis, cases with mild forms of persistent $A R$, and control or healthy group. We obtained biopsies of nasal inferior turbinate mucosa from all participants. Expression of AMCase and IL-8 mRNAs were evaluated by real-time polymerase chain reaction (PCR). The serum levels of AMCase and IL-8 were determined by ELISA. The number of eosinophils per field, blood eosinophils, total serum IgE levels, and specific serum IgE levels were measured. Patients' clinical manifestations were assessed by total nasal syndrome score (TNSS).

Results: Expression of AMCase and IL-8 in patients with moderate and severe perineal allergic rhinitis were significantly elevated compared to the control group and patients with mild persistent allergic rhinitis. Serum levels of AMCase and IL-8 were associated with specific IgE, nasal eosinophil count, and TNSS.

Conclusions: According to the results of this study, there might be a relationship between the expression of AMCase and IL-8 in nasal turbinate mucosa and the severity of allergic rhinitis.
\end{abstract}

Key words: allergic rhinitis, AMCase, IL-8, inferior turbinate, nasal mucosa, gene expression.

(Cent Eur J Immunol 2020; 45 (3): 294-300)

\section{Introduction}

Epidemiologic studies indicate that the universal frequency of allergic rhinitis (AR) is growing and affecting 400 million people [1]. AR is described as "indications of sneezing, nasal pruritus, airflow congestion, and mostly clear nasal discharge induced by IgE-mediated reactions against inhaled allergens", and includes mucosal swelling driven by type 2 helper T (Th2) cells [2].

Previously, it was recognized that acidic mammalian chitinase (AMCase) is a critical element of IL-13-stimulated reaction in Th2-dominated disorder in both human and animal asthma models [3-5]. Furthermore, it was thought that chitinase from house dust mite, such as Der- matophagoides pteronyssinus (D. pteronyssinus) and Dermatophagoides farinae (D. farinae), present a high potential of binding to IgE in atopic human sera [6], and are the primary allergens for humans and dogs with house dust mite hypersensitivity [7]. The production of cytokines and chemokines, such as IL-8, which is a member of the CXC chemokine family, is heightened by the airway epithelial cells in response to environmental elements, including pollens, cigarette smoke, air contaminations, and microorganisms with their secreted extracellular proteins [8-10]. IL-8 has been considered a crucial factor in airway inflammatory disorder $[11,12]$. Moreover, high levels of IL-8 have been detected in the bronchoalveolar lavage fluid of patients with airway swelling disorders [13].

Correspondence: Sayed Asadollah Amini, Clinical Biochemistry Research Center, Shahrekord University of Medical Sciences, 8815713471 Shahrekord, Iran, e-mail: s.amini1362@yahoo.com

Submitted: 17.12.2017; Accepted: 6.05.2018 
Therefore, the present study was undertaken to:

Investigate the human serum expression levels of AMCase and IL-8 in patients with moderate/severe persistent allergic rhinitis (M/S PAR) compared to patients with mild persistent allergic rhinitis (M PAR) and the control group.

Evaluate mRNA expression of AMCase and IL-8 in human inferior turbinate mucosa in patients with M/S PAR compared to its expression in patients with M PAR and the control group.

Determine the correlation between serum levels of AMCase and IL-8 as well as the correlation of each serum levels with various pathological parameters.

\section{Material and methods}

\section{Subjects}

Patients were administered into three groups: M/S PAR $(n=30), \operatorname{M~PAR~}(n=30)$, and control $(n=30)$ groups. The selected patients presented with no history of nasal infection, allergy, smoking, and ongoing drug treatment. During surgery, inferior turbinate mucosal biopsies were obtained from all included patients. The diagnosis of AR was established on the basis of medical history and symptoms (i.e., sneezing, rhinorrhea, and nasal congestion occurring most days) lasting for at least 2 years, positive skin test results, and positive specific IgE ELISA test for house dust mite (Astra Biotech GmbH, Berlin, Germany). AR patients were classified as mild or moderate/severe persistent according to allergic rhinitis and its impact on asthma criteria [14]. Exclusion criteria consisted of current smoking status, medical history of asthma and recurrent infections of respiratory tract, and the use of anti-allergic drugs within 4 weeks prior the study. Before tissue specimens were obtained, all the protocols have been approved by the institutional review board at our institution, and all participants have signed a written informed consent. Venous blood samples were obtained from patients. Blood drawing, handling, and storage were performed by the same researchers. Some of the tissues were immediately frozen in liquid nitrogen and stored at -70 centigrade for the extraction of RNA, and some samples were paraphined for hematoxylin and eosin (H\&E) staining. Four-millimeter punch nasal biopsies were collected from inferior turbinate of both patients and control groups. Biopsy specimens were submerged immediately in $10 \%$ buffered formalin and prepared for paraffin embedding. Paraffin blocks were cut into sections of four microns thick, which were mounted on glass slides. They were prepared for routine H\&E staining for conventional histopathology (Table 1).

\section{Real-time polymerase chain reaction analysis of AMCase and IL-8}

The total RNA was isolated from nasal tissue using Biozol reagent (BioFlux, Japan). The quantity and qual- ity of RNAs were measured by spectrophotometry using wavelength absorption ratio (260/280). Some RNA samples $(1 \mathrm{mg} / \mathrm{ml})$ were coupled with DNase $1(10 \mathrm{u} / \mathrm{ml})$ for $30 \mathrm{~min}$ to avoid DNA contamination. Following the second RNA extraction, cDNA was produced in a $20 \mathrm{ml}$ reaction tube from $1 \mathrm{mg}$ total RNA (Thermo Fisher Scientific, Vilnius, Lithuania) with a random primer. The reactions were incubated at $25^{\circ} \mathrm{C}$ for $5 \mathrm{~min}, 42^{\circ} \mathrm{C}$ for $1 \mathrm{~h}$, and $72^{\circ} \mathrm{C}$ for $5 \mathrm{~min}$. The primer sequences, which were used for each gene included in the study are shown in Table 1 . $\beta$-actin was used as the internal control. Expressions were determined by quantitative real-time polymerase chain reaction (PCR) using SYBR Green PCR master mix (Takara, Kyoto, Japan). A standard curve was established, and efficiency was determined for each set of primers. The determined efficiency was typically above $90 \%$. The data were expressed as fold changes, using comparative cycle threshold ( $\Delta \Delta \mathrm{ct}$ method). Relative gene expression was defined by the $2-\Delta \Delta$ ct method.

\section{Assessment of AMCase and IL-8 levels by ELISA}

AMCase and IL-8 levels in serum of patients with M/S PAR, patients with M PAR, and controls were measured using ELISA as per the manufacturer's protocol (Abnova, Taipei, Taiwan). Data were expressed as $\mathrm{ng} / \mathrm{ml}$ protein. Protein concentration was determined with the Starsat ELISA reader (Awarness, Phoenix, AZ, USA).

\section{Statistical analyses}

Statistical analyses were carried out using SPSS for Windows (IBM SPSS Statistics, IBM). One-way ANOVA, along with post-hoc Tukey's test was performed in order to evaluate whether the values obtained by real-time PCR and ELISA differ among the nasal mucosa of healthy controls and patients with mild and M/S PAR. Student's $t$-test was used to analyze the differences in specific IgE between M/S PAR and mild PAR. Correlations were assessed with Pearson's correlation test. A value of $p<0.05$ was considered as statistically significant.

Table 1. Sequences of PCR primers

\begin{tabular}{ll}
\hline$\beta$-actin & Forward primer: \\
& 5-AGC GAG CAT CCC CCA AAG TT-3 \\
& Reverse primer: \\
& 5-GGG CAC GAA GGC TCA TCA TT-3 \\
\hline \multirow{2}{*}{ MCase } & Forward primer: \\
& 5-TGTCTGTCGGAGGATGGAACT-3 \\
& Reverse primer: \\
& 5-TCTGGGTGTTGGAGGCTATCTT-3 \\
\hline \multirow{2}{*}{ IL-8 } & Forward primer: \\
& 5-TGAAGGACATGGCTTAGAAGTG-3 \\
& Reverse primer: 5-GGTGCAAGGGTCACAGTGTT-3 \\
\hline &
\end{tabular}


Table 2. Demographic characteristics of participants

\begin{tabular}{lccc}
\hline Variable & Healthy control & M PAR & M/S PAR \\
\hline Gender, male/female & $18 / 12$ & $20 / 10$ & $22 / 8$ \\
\hline Age & $28.5 \pm 6.42$ & $26.03 \pm 4.03$ & $26.7 \pm 4.87$ \\
\hline SIgE for house dust mite $(\mathrm{IU} / \mathrm{ml})$ & 0 & $0.51 \pm 0.15$ & $4.57 \pm 5.56$ \\
\hline TIgE $(\mathrm{IU} / \mathrm{ml})$ & $82.63 \pm 43.88$ & $236.3 \pm 33.24$ & $1061.1 \pm 88.41$ \\
\hline Blood eosinophil counts & $74.5 \pm 40.02$ & $255.7 \pm 70.96$ & $607.27 \pm 117.37$ \\
\hline Degree of eosinophil infiltration into nasal mucosa & $0.5 \pm 0.5$ & $1.7 \pm 0.59$ & $3.63 \pm 1.88$ \\
\hline Disease duration in years & 0 & $15.63 \pm 2.09$ & $17.17 \pm 4.39$ \\
\hline
\end{tabular}
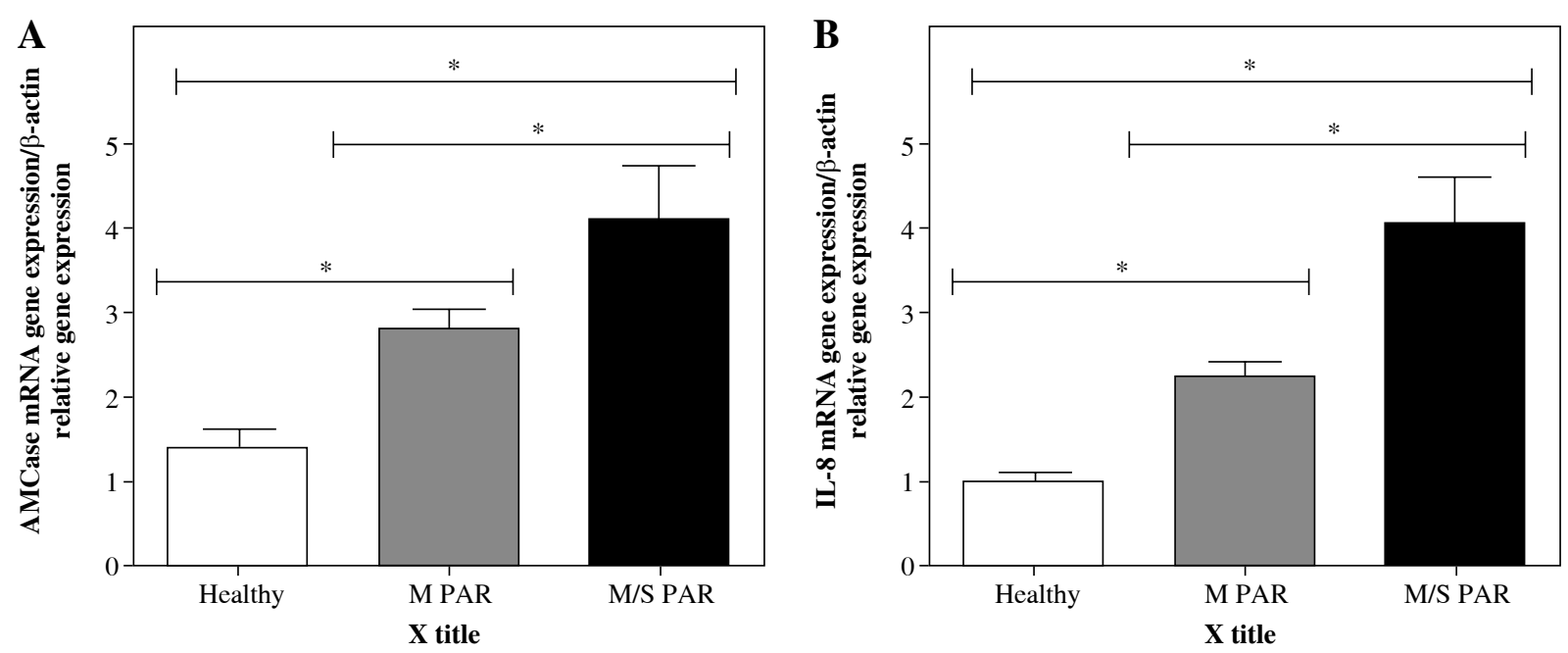

Fig. 1. Real-time PCR analysis of AMCase (A) and IL-8 (B). mRNA in inferior turbinate nasal mucosal tissue from controls and patients with M PAR and M/S PAR. * indicates significant difference in the expression levels of AMCase and IL-8 among normal, mild, and moderate/severe persistent allergic nasal mucosa $(p<0.05)$. Results are shown as mean \pm SD (Table 3)

Table 3. Comparison of baseline characteristics, AMCase gene expression, IL-8 gene expression, serum levels of AMCase, and IL-8 among control group, patients with M PAR, and patients with M/S PAR

\begin{tabular}{lccc}
\hline Variable & Control & M PAR & M/S PAR \\
\hline AMCase gene expression & $1.4 \pm 1.15$ & $2.81 \pm 1.2$ & $4.13 \pm 3.32$ \\
\hline IL-8 gene expression & $1.01 \pm 0.53$ & $2.25 \pm 0.95$ & $4.04 \pm 3.06$ \\
\hline Serum AMCase level & $17.89 \pm 2.98$ & $19.97 \pm 3.65$ & $23.79 \pm 2.78$ \\
\hline Serum IL-8 level & $10.1 \pm 3.21$ & $14.41 \pm 2.22$ & $26.08 \pm 3.35$ \\
\hline Results are shown as mean $\pm S D$ & & &
\end{tabular}

\section{Results}

\section{Clinical characteristics of participants}

The characteristics of patients with persistent allergic rhinitis and healthy controls are demonstrated in Table 2.
As shown in Table 2, age was similarly distributed among the healthy controls and the patients with PAR. The duration of disease did not significantly differ between the groups of patients with $\mathrm{M}$ and M/S PAR, and serum total IgE levels were higher in patients with $M$ and M/S PAR than in individuals with healthy nasal mucosa.

\section{Gene expression levels of AMCase and IL-8 in nasal mucosa}

The gene expression levels of AMCase and IL-8 were determined in healthy, M, and M/S PAR nasal mucosa samples using real-time PCR. As shown in Figure 1 and Table 3, the expression levels of AMCase and IL-8 presented significant increase in nasal mucosa of patients with M/S PAR compared to nasal mucosa of patients with M PAR and the controls. Similarly, the gene expressions of AMCase and IL- 8 were statistically different between M PAR and control groups. 

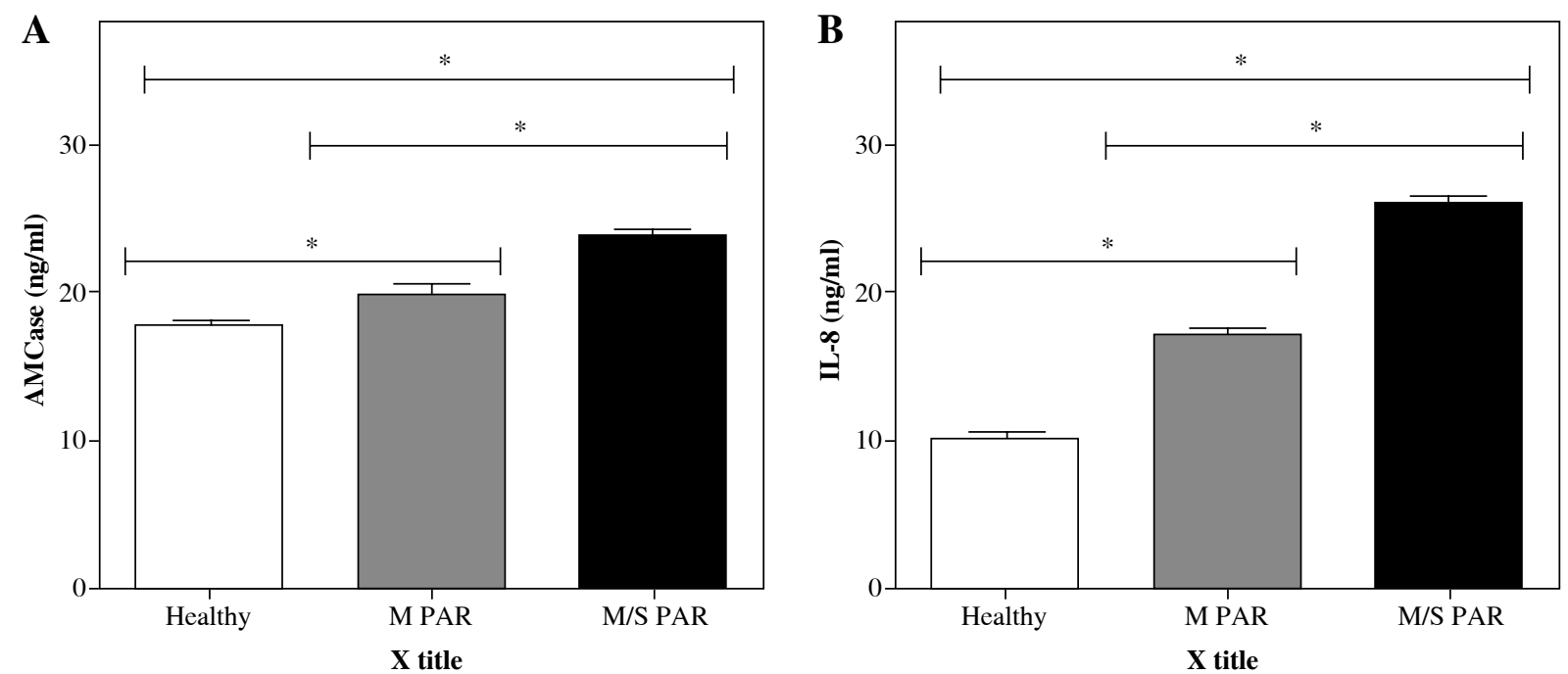

Fig. 2. Comparison between serum levels of AMCase (A) and IL-8 (B) in controls and patients with M PAR and M/S PAR. * indicates a significant difference in the expression levels of AMCase and IL-8 among normal, M PAR, and M/S PAR nasal mucosa $(p<0.05)$. Results are shown as mean \pm SD

\section{Serum levels of AMCase and IL-8 in patients with M/S PAR, M PAR, and healthy controls}

As shown in Figure 2 and Table 3, AMCase and IL-8 levels $(\mathrm{ng} / \mathrm{ml})$ were measured in the serum obtained from patients with M/S PAR, M PAR, and healthy controls. Significantly higher levels of AMCase and IL-8 were found in serum obtained from patients with M/S PAR compared to healthy participants $(p<0.05)$ and patients with M PAR. Likewise, the differences between the patients with $M$ PAR and healthy controls in terms of levels of AMCase and IL-8 were statistically significant $(p<0.05)$.

\section{Associations between serum levels of AMCase with immunopathogenesis of M/S PAR}

As shown in Figure 3, there were considerable associations between serum AMCase levels and TNSS $(R=0.576$, $p<0.05$; Fig. 3A), degree of eosinophil infiltration to nasal $(R=0.393, p<0.05$; Fig. 3B $)$, and $\operatorname{SIgE}(R=0.524$, $p<0.05$; Fig. 3C). On the other hand, serum AMCase levels did not correlate with blood eosinophil counts $(R=0.178, p>0.05$; Fig. 3D) or TIgE $(R=0.277$, $p>0.05$; Fig. 3E). Moreover, the correlation between serum AMCase levels and serum IL-8 levels was statistically significant $(R=0.525, p<0.05$; Fig. 3F $)$.

\section{Associations between serum levels of IL-8 with immunopathogenesis of M/S PAR}

As shown in Figure 4, the correlations between serum IL-8 levels and TNSS $(R=0.428, p<0.05$; Fig. 4A), degree of eosinophil infiltration to nasal $(R=0.547$, $p<0.05$; Fig. 4B), and $\operatorname{SIgE}(R=0.473, p<0.05$; Fig. 4C $)$ were statistically visible. In contrast, there was no association between serum IL-8 levels with blood eosinophil counts $(R=0.114, p>0.05$; Fig. 4D) and $\operatorname{TIgE}(R=0.267$, $p>0.05$; Fig. 4E).

\section{Discussion}

In this study, gene expressions of AMCase and IL-8 in the human nasal mucosa of inferior turbinate as well as the serum levels were investigated. Although local and serum production of AMCase and IL-8 in AR have previously been reported [15-18], this was the first study (as far as the authors of the present study are concerned) conducted on the correlation among AMCase and IL-8 with disease parameters, including disease severity and clinical characteristics. The findings of the present study showed that there was an observable increase in serum levels and gene expression of AMCase and IL-8 in patients with M/S PAR compared to patients with M PAR and the controls. Similar to the data presented here, Cho et al. [15] reported that production of AMCase increased in patients with AR compared to healthy controls. Moreover, Gosset et al. [19] stated that production of IL- 8 in patients with AR raised compared to healthy controls. On the contrary, the results obtained from the present study was rejected by Dassonville [20] and Dasilva [21]. Data on cellular spreading is required to disclose practical characteristics of AMCase. The gene expression of AMCase has been recognized by northern blotting analysis in human submandibular gland and stomach [22]. This pattern of spreading suggested that AMCase takes on a significant role in the digestion of ingested chitin-coated animals and plants rather than being a mere defense system [22]. Nevertheless, it is possible 

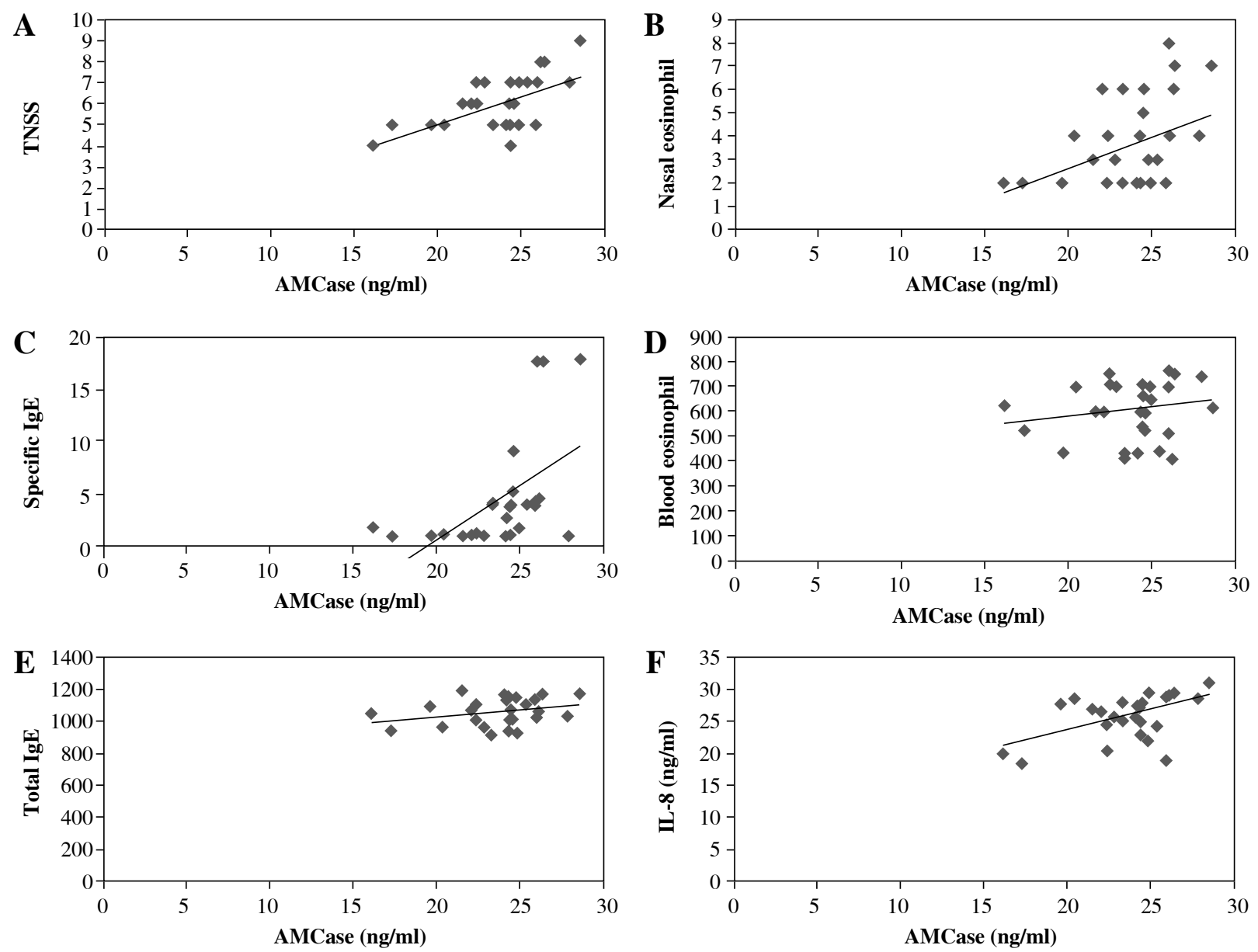

Fig. 3. Correlations between serum levels of AMCase and total nasal score syndrome (TNSS) (A), nasal eosinophil count (B), specific $\operatorname{IgE}(\mathrm{SIgE})(\mathbf{C})$, blood eosinophil count $(\mathbf{D})$, total $\operatorname{IgE}(\mathrm{TIgE})(\mathbf{E})$, and serum IL-8 levels $(\mathbf{F})$ in patients with moderate/severe persistent allergic rhinitis (M/S PAR)

that AMCase participates in defense against chitin coating pathogens, such as fungi. Overdijk et al. [23] suggested that the attendance of chitinase in human serum may be due to a function of AMCase in the defense against chitinous pathogens, such as fungi. This suggestion is supported by the observation that a systemic infection with Aspergillus Fumigates results in increasing chitinase levels and this, in turn, can be inhibited by antifungal treatment [24]. However, a great number of macromolecules, consisting of mucine and antibacterial substances, are secreted by submucosal glands and epithelial cells in nasal mucosa. Cho et al. [15] reported that AMCase was noticeably produced in the epithelial cells and submucosal glands in healthy nasal mucosa, indicating its participation in defense against chitin-containing pathogens. Moreover, it may play a role in allergic reaction similar to other inflammatory mediators in allergic rhinitis, due to its up-regulation of allergic conditions. One of the strengths of this study was that the correlations among AMCase and IL-8 with clinical char- acteristics were analyzed. It was found that serum levels of AMCase and IL-8 were positively associated with SIgE and the number of infiltrating eosinophils to nasal mucosa. Nasal eosinophils could induce inflammation, which cause nasal hyperactivity. Interestingly, there were visible correlations between AMCase and IL- 8 with TNSS; therefore, the present data support the concept that AMCase and IL-8 are associated with disease severity. Serum levels of AMCase are associated with congestion and sneezing. In the present study, there was a correlation between IL-8 and sneezing; however, the correlations between both AMCase and IL-8 with rhinorrhea were not statistically significant. In addition, there was a positive correlation between serum levels of AMCase and IL-8, indicating that up-regulation of AMCase was associated with up-regulation of IL-8. Another noteworthy finding was that serum levels of AMCase and IL-8 were not correlated with blood eosinophil count, suggesting that it was more reasonable to estimate the level of eosinophils in tissue than in blood. 

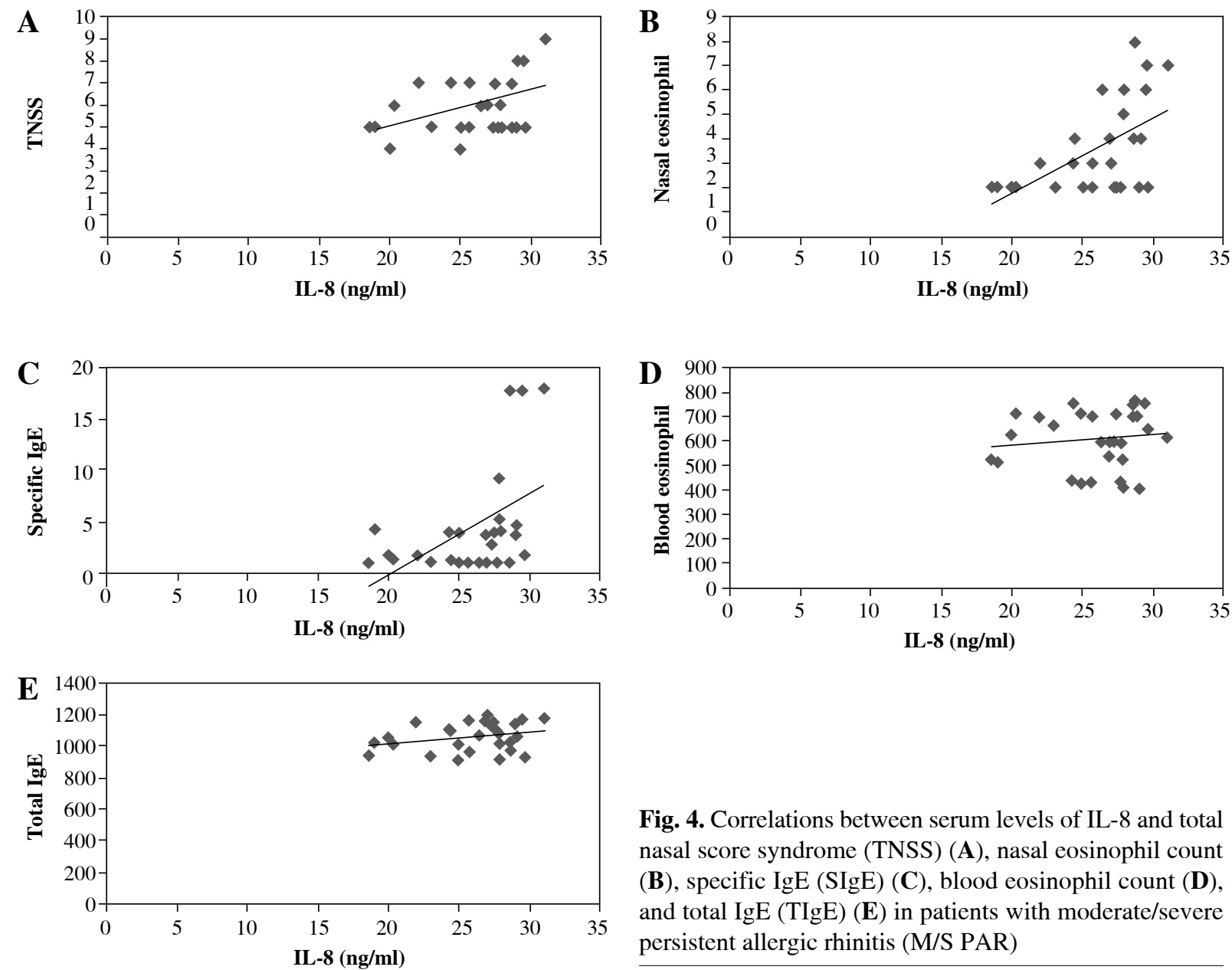

Fig. 4. Correlations between serum levels of IL-8 and total nasal score syndrome (TNSS) (A), nasal eosinophil count (B), specific IgE (SIgE) (C), blood eosinophil count (D), and total $\operatorname{IgE}(\mathrm{TIgE})(\mathbf{E})$ in patients with moderate/severe persistent allergic rhinitis (M/S PAR)

\section{Conclusions}

The present study showed that disease parameters are associated with up-regulation of AMCase and IL-8.

\section{Acknowledgments}

This work has been obtained from MSc thesis of Mr. Hamid Reza Sadeghi. Additionally, we wish to acknowledge the financial support of research council of Shahrekord University of Medical Sciences (No. 1918-87-011392).

The authors declare no conflict of interest.

\section{References}

1. Baena-Cagnani CE, Canonica GW, Helal MZ, et al. (2015): The international survey on the management of allergic rhinitis by physicians and patients. World Allergy Organ J 8: 10.

2. Solomon CG, Wheatley LM, Togias A (2015): Allergic Rhinitis. N Engl J Med 372: 456-463.

3. Zhu Z, Zheng T, Homer RJ, et al. (2004): Acidic mammalian chitinase in asthmatic Th2 inflammation and IL-13 pathway activation. Science 304: 1678-1682.

4. Elias JA, Homer RJ, Hamid Q, Lee CG (2005): Chitinases and chitinase-like proteins in T $\mathrm{H} 2$ inflammation and asthma. J Allergy Clin Immunol 116: 497-500.

5. Wills-Karp M, Karp CL (2004): Chitin checking-novel insights into asthma. N Engl J Med 351: 1455-1457.

6. O'Neil SE, Heinrich TK, Hales BJ, et al. (2006): The chitinase allergens Der p 15 and Der p 18 from Dermatophagoides pteronyssinus. Clin Exp Allergy 36: 831-839.

7. Weber E, Hunter S, Stedman K, et al. (2003): Identification, characterization, and cloning of a complementary DNA encoding a 60-kd house dust mite allergen (Der f 18) for human beings and dogs. J Allergy Clin Immunol 112: 79-86.

8. Matsui EC, Hansel NN, McCormack MC, et al. (2008): Asth$\mathrm{ma}$ in the inner city and the indoor environment. Immunol Allergy Clin North Am 28: 665-686.

9. Mio T, Romberger DJ, Thompson AB, et al. (1997): Cigarette smoke induces interleukin-8 release from human bronchial epithelial cells. Am J Respir Crit Care Med 155: 1770-1776.

10. Hasan Bayram, Jagdish L, Devalia, et al. Davies (1998): The effect of diesel exhaust particles on cell function and release of inflammatory mediators from human bronchial epithelial cells in vitro. Am J Respir Cell Mol Biol 18: 441-448. 
11. Sohn MH, Lee KE, Choi SY, et al. (2005): Effect of Mycoplasma pneumoniae lysate on interleukin-8 gene expression in human respiratory epithelial cells. Chest 128: 322-326.

12. Lee K, Kim J, Jeong K, et al. (2007): Regulation of German cockroach extract-induced IL-8 expression in human airway epithelial cells. Clin Exp Allergy 37: 1364-1373.

13. Ordonez CL, Shaughnessy TE, Matthay MA, Fahy JV (2000): Increased neutrophil numbers and IL-8 levels in airway secretions in acute severe asthma: clinical and biologic significance. Am J Respir Crit Care Med 161: 1185-1190.

14. Jun YJ, Park SJ, Hwang JW, et al. (2014): Differential expression of $11 \beta$-hydroxysteroid dehydrogenase type 1 and 2 in mild and moderate/severe persistent allergic nasal mucosa and regulation of their expression by Th2 cytokines. Clin Exp Allergy 44: 197-211.

15. Cho WS, Kim TH, Lee HM, et al. (2010): Increased expression of acidic mammalian chitinase and chitotriosidase in the nasal mucosa of patients with allergic rhinitis. Laryngoscope 120: 870-875.

16. Nikota JK, Botelho FM, Bauer CMT, et al. (2011): Differential expression and function of breast regression protein 39 (BRP-39) in murine models of subacute cigarette smoke exposure and allergic airway inflammation. Respir Res 12: 39.

17. Kovalhuk L, Rosário NA, Carvalho A (2001): Inflammatory mediators, cell counts in nasal lavage and computed tomography of the paranasal sinuses in atopic children. J Pediatr (Rio J) 77: 271-278.

18. KleinJan A, Dijkstra MD, Boksa SS, et al. (1999): Increase in IL-8, IL-10, IL-13, and RANTES mRNA levels (in situ hybridization) in the nasal mucosa after nasal allergen provocation. J Allergy Clin Immunol 103: 441-450.

19. Gosset P, Malaquin F, Durieu J, et al. (1997): Interleukin-8 secretion in patients with allergic rhinitis after an allergen challenge: interleukin- 8 is not the main chemotactic factor present in nasal lavages. Clin Exp Allergy 27: 379-388.

20. Dassonville C, Bonfils P, Momas I, Seta N (2007): Nasal inflammation induced by a common cold: comparison between controls and patients with nasal polyposis under topical steroid therapy. Acta Otorhinolaryngol Ital 27: 78.

21. da Silva TM, Guimarăes RES, Nascimento E, et al. (2009): RT-PCR cytokine study in patients with allergic rhinitis. Braz J Otorhinolaryngol 75: 24-29.

22. Boot RG, Bussink AP, Verhoek M, et al. (2005): Marked differences in tissue-specific expression of chitinases in mouse and man. J Histochem Cytochem 53: 1283-1292.

23. Overdijk B, Van Steijn GJ (1994): Human serum contains a chitinase: identification of an enzyme, formerly described as 4-methylumbelliferyl-tetra-N-acetylchitotetraoside hydrolase (MU-TACT hydrolase). Glycobiology 4: 797-803.

24. Overdijk B, Van Steijn GJ, Odds FC (1996): Chitinase levels in guinea pig blood are increased after systemic infection with Aspergillus fumigatus. Glycobiology 6: 627-634. 